\title{
Development of a gaseous proton-recoil detector for fission cross section measurements below $1 \mathrm{MeV}$ neutron energy
}

\author{
P. Marini ${ }^{1}$, L. Mathieu ${ }^{1 a}$, M. Aïche ${ }^{1}$, S. Czajkowski ${ }^{1}$, B. Jurado ${ }^{1}$, I. Tsekhanovich ${ }^{1}$ \\ ${ }^{1}$ Centre d'Etudes Nucléaires de Bordeaux Gradignan, 19, Chemin du Solarium, 33175 GRADIGNAN, France
}

\begin{abstract}
The elastic H(n,p) reaction is sometimes used to measure neutron flux, in order to produce high precision measurements. The use of this technique is not straightforward to use below incident neutron energy of $1 \mathrm{MeV}$, due to a high background in the detected proton spectrum. Experiments have been carried out at the AIFIRA facility to investigate such background and determine its origin and components. Based on these investigations, a gaseous proton-recoil detector has been designed, with a reduced low energy background.
\end{abstract}

\section{Introduction}

The development of Generation IV nuclear system relies on accurate system modelling, to determine their behaviour and its evolution with time, irradiation and/or in incidental/accidental situations. These simulations require high quality physical models and nuclear data to meet the requested precision [1]. Despite having been studied for decades, large discrepancies and uncertainties exist on nuclear data in the actinide region [2]. In particular, the knowledge of fission cross sections is of primary importance for determining the nuclear core behaviour or waste incineration capabilities.

The neutron flux maximum in a fast reactor, such as the Sodium Fast Reactor, is located around $100 \mathrm{keV}$ [3]. At these energies, cross sections of non-fissile isotopes are quite low. Nevertheless, some of them are not negligible (for instance ${ }^{240,242} \mathrm{Pu}(\mathrm{n}, \mathrm{f})$ ) and may have an impact on the core behaviour. Strong requests are then addressed to improve the fission cross section in this energy range.

Cross section measurements are often normalized to standard reactions such as ${ }^{235} \mathrm{U}(\mathrm{n}, \mathrm{f}),{ }^{238} \mathrm{U}(\mathrm{n}, \mathrm{f})$ or ${ }^{237} \mathrm{~Np}(\mathrm{n}, \mathrm{f})$. Since the systematic normalization of the measurements to the mentioned cross sections introduces a strong correlation between the different results, independent measurements are carried out relative to the $\mathrm{H}(\mathrm{n}, \mathrm{p})$ scattering. In this method, the neutron flux is converted into a proton flux by an H-rich foil. As a charged particle, proton can be easily detected, for instance in a silicon detector. Different types of proton-recoil detector exist, depending on the type of experiment, the facility environment (neutron beam vs. isotropic neutron source), the neutron energy range or the target accuracy [4-13].

\footnotetext{
${ }^{a}$ Corresponding author: mathieu@cenbg.in2p3.fr
} 


\section{Proton-recoil usual technique}

The ACEN group of the Centre d'Etudes Nucléaires de Bordeaux Gradignan (CENBG) has developed in the 2000's its own proton-recoil setup to perform accurate measurements in the MeV energy range [4]. It consists of a polypropylene foil with a thickness ranging from $20 \mu \mathrm{m}$ to $50 \mu \mathrm{m}$, for neutron energy of $2 \mathrm{MeV}$ to $5 \mathrm{MeV}$ respectively. A Silicon telescope is placed at $7.5 \mathrm{~cm}$ downstream on the beam axis to detect protons scattered at very low scattering angle. These protons have kinetic energies close to the incident neutron energy. Nearly mono-energetic incident neutrons produce a clear proton peak in the proton spectrum, with a very good signal-to-background ratio. A collimator placed in front of the $\mathrm{Si}$ detector is used to accurately define the detection efficiency. Fission cross sections were measured with this experimental setup, with final uncertainties as low as 3-4\% [4].

In 2012, the setup was adapted to neutron energy around $1 \mathrm{MeV}$ for measurement at the BruyèresLe-Chatel 4MV Van-de-Graaff accelerator. The silicon telescope was replaced by a single layer silicon detector and the polypropylene foil thickness was reduced to $4 \mu \mathrm{m}$. At these energies, the signal-to-background ratio at the proton peak is strongly reduced, due to a strong background at lower energies. Such background prevents accurate neutron flux measurements at neutron energy of few hundreds keV.

Measurement was performed in 2014 at the AIFIRA facility to investigate the background and how to reduce it. Several runs were performed with a $50 \mu \mathrm{m}$-thick silicon detector, for incident neutron energies ranging from $1.6 \mathrm{MeV}$ down to $500 \mathrm{keV}$. The proton spectra obtained are shown on Figure 1. As the incident energy decreases, the proton peak shifts toward lower energies and is progressively hidden by a strong background. At $\mathrm{E}_{\mathrm{n}}=700 \mathrm{keV}$, the proton peak is still visible but its integral (used to infer the neutron flux) cannot be determined with an accuracy better than $30 \%$. At $\mathrm{E}_{\mathrm{n}}$ $=500 \mathrm{keV}$, the proton peak is barely visible.
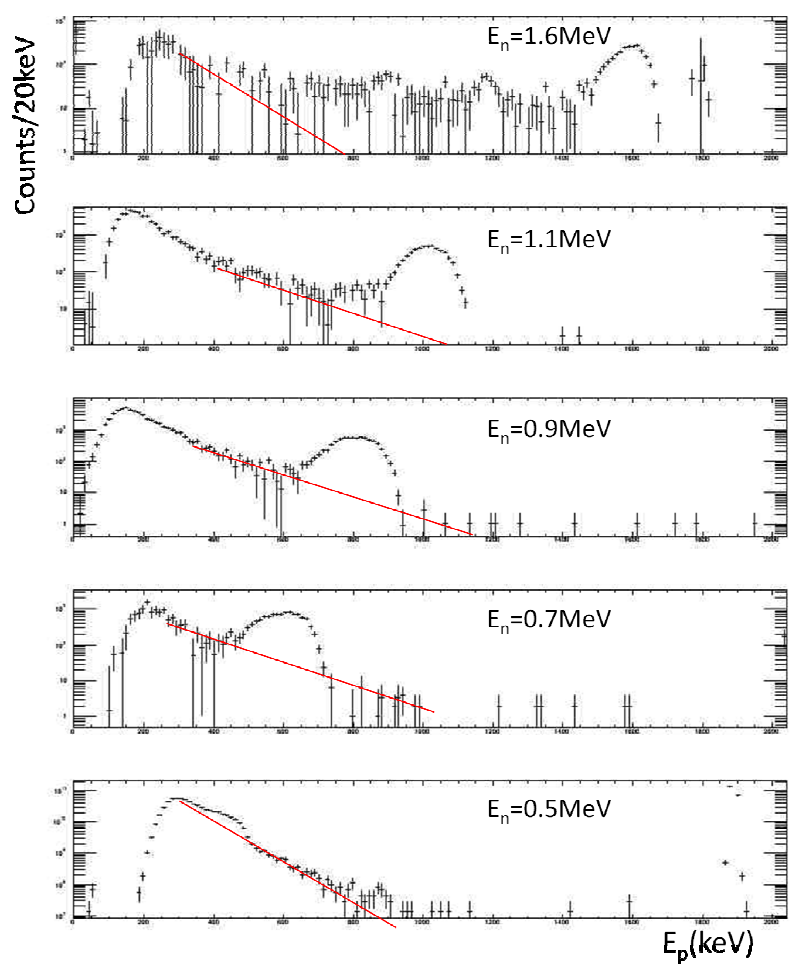

Figure 1. Proton spectra for different incident neutron energies (from $1.6 \mathrm{MeV}$ to $0.5 \mathrm{MeV}$ ). The proton peak can be easily distinguished from the background for $\mathrm{E}_{\mathrm{n}}$ greater than $1 \mathrm{MeV}$. For lower energies, the signal-tobackground ratio is strongly reduced, preventing any accurate determination of the neutron flux. 
Simulations were performed with the MCNP code to reproduce these results. Several hypothesises were tested to reproduce the exponential shaped background. The simulations show that low energy $\gamma$ rays (few hundreds of $\mathrm{keV}$ ) can produce electrons by Compton scattering, which deposit their energy in the silicon detector. Contrarily to protons, which interact in the first few microns of the detectors (range of 18, 9 and $2 \mu \mathrm{m}$ at 1.1, 0.7 and $0.2 \mathrm{MeV}$ respectively), the electrons interact with the whole detector thickness. Even if they deposit only a small fraction of their energy, a $50 \mu \mathrm{m}$-thick silicon detector is then not suitable to discriminate low energy protons in a not negligible electron flux.
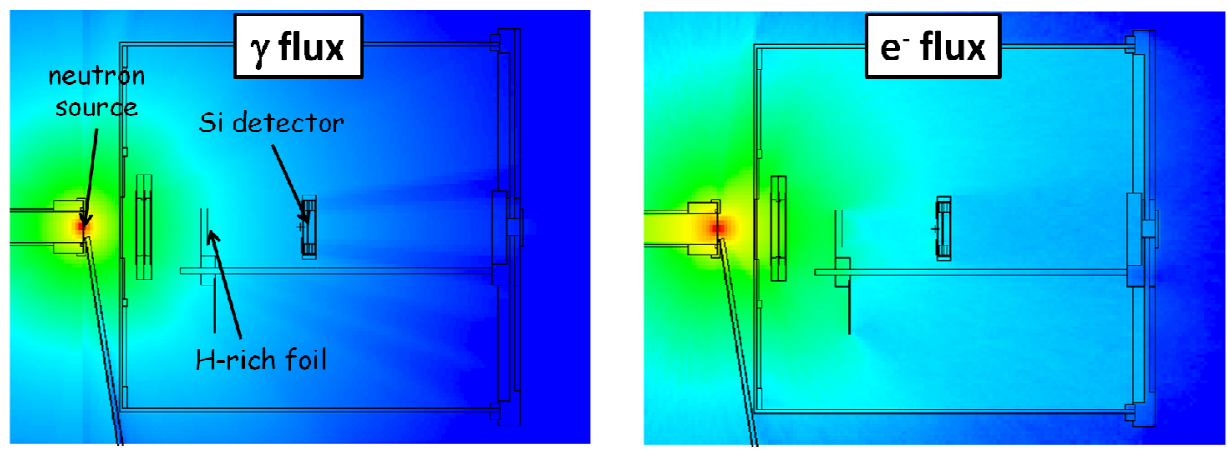

Figure 2. Simulations of $\gamma$-ray and electron flux in the experimental setup, with a $\gamma$-ray source of $200 \mathrm{keV}$ located at the neutron production target. Electrons produced by Compton scattering are present in the whole setup and some deposit their energy in the Si detector.

\section{DPR Design}

Thinner Si detectors, with thickness down to few $\mu \mathrm{m}$, would reduce the electron noise contribution. However this solution is not straightforward. The ACEN group decided to design a gaseous protonrecoil detector: the protons emitted by an $\mathrm{H}$-rich foil are detected in an ionization chamber, filled with a $\mathrm{CF}_{4}$ gas and segmented into two chambers $\Delta \mathrm{E}$ and $\mathrm{E}$ separated by a collimator. The gas pressure is adjusted to the proton energy range in order to fit with the detector length. Electron energy deposition in such a low density material is very low, leading to a strongly reduced background. The coincidence between the two ionization chambers $\Delta \mathrm{E}$ and $\mathrm{E}$ also significantly reduces parasitic recoil protons coming from other sources than the H-rich foil. A scheme of this detector is presented in Figure 3.

To perform high precision measurements, the detector efficiency has to be very well defined. The collimator between the two ionization chambers and a second collimator after the H-rich foil allow us to determine a precise geometrical efficiency. $\mathrm{Th} \mathrm{CF}_{4}$ gas and ceramic materials used for the structures were chosen for very low or absent hydrogen content, so that no recoil protons can be emitted from the gas or the frame which would induce systematic errors or background.

For low incident neutron energies, the deposited energy in the gas is quite low. The generated primary electrons are collected and amplified by a Micromegas detector. To reduce electronic background, the detector is segmented into 64 pads. This will also make a crude track analysis possible, to reject false coincidence with abnormal trajectories (for instance not starting from the $\mathrm{H}$ rich foil). Moreover, the choice of the fast $\mathrm{CF}_{4}$ gas (i.e high electron mobility) reduces the pile-up of the signal with a possible background noise. 


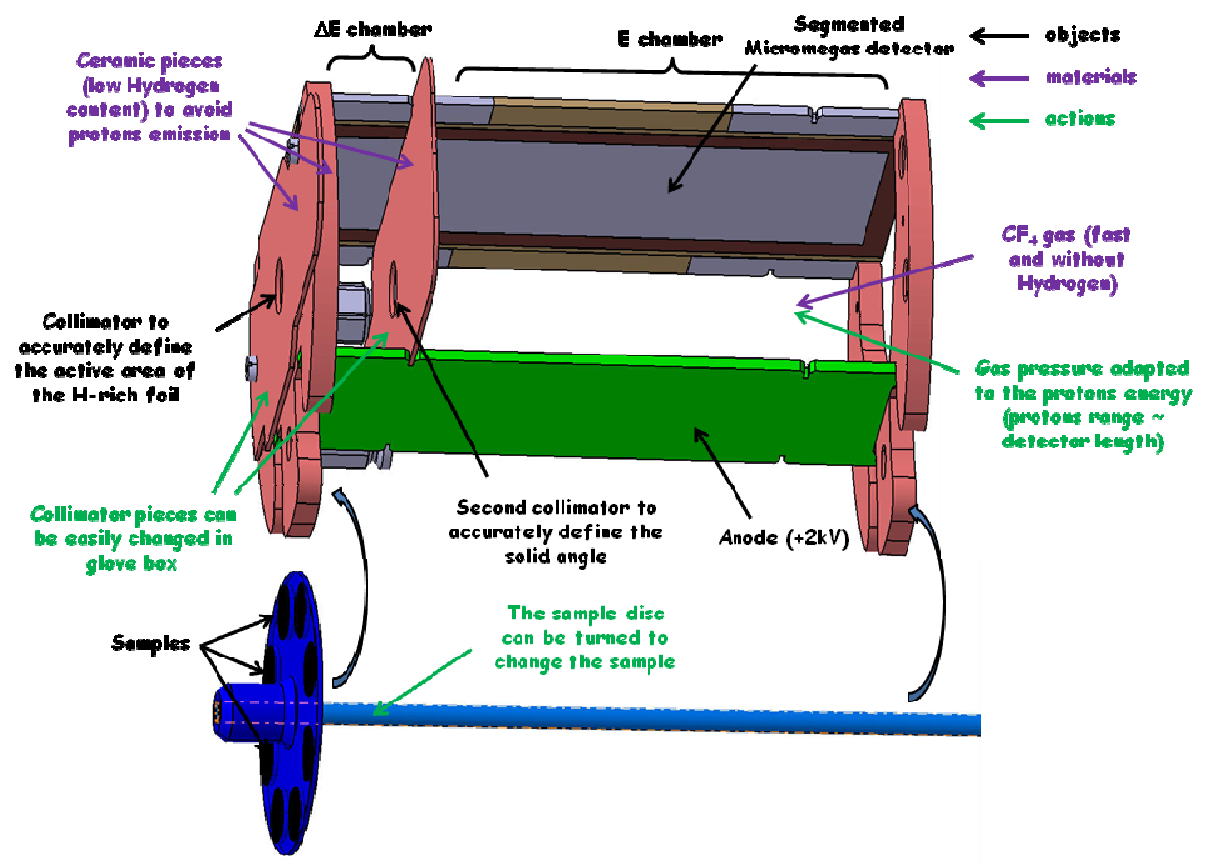

Figure 3. Scheme of the gaseous proton-recoil detector. For representation purpose, the sample disc has been separated from the proton-recoil detector.

To reduce the proton energy loss in the H-foil itself, several samples of different thicknesses (from $1 \mu \mathrm{m}$ to $10 \mu \mathrm{m}$ ) are mounted on a disc (on the bottom of Figure 3). Thinnest samples are evaporated tristearine, while the thickest ones are polypropylene films. An empty hole is also available. The disc can be turned to select the sample thickness adapted to the incident neutron energy, or to perform background measurement without sample (empty hole).

To gaseous proton-recoil detector has been designed by the CENBG and will be manufactured in late 2015 . The electronic connections will be set in early 2016, for an operational release planned in the first half of 2016.

\section{Conclusions}

Neutron flux measurements via the elastic scattering $H(n, p)$ reaction is challenging for neutron energies below $1 \mathrm{MeV}$. Gamma rays produced by the neutron production target generate electrons which induce a strong background in the proton detector, preventing an accurate determination of the number of recoil protons.

A new detector based on the proton detection in a gas is being designed at the CENBG. Its low electron sensitivity is suited for neutron energies below $1 \mathrm{MeV}$ in a gammas and electrons dense environment (i.e Van-de-Graaff type accelerator). The commissioning will take place in the first half of 2016.

\section{References}

1. G. Aliberti, G. Palmiotti, M. Salvatores, T. Kim, T. Taiwo, M. Anitescu, I. Kodeli, E. Sartori, J. Bosq, J. Tommasi, Ann. Nucl. Ener. 33, 700 (2006)

2. M. Chadwick, M. Herman, P. Oblozinsky, M. Dunn, Y. Danon, A. Kahler, D. Smith, B. Pritychenko, G. Arbanas, R. Arcillas et al., Nucl. Data Sheets 112, 2887 (2011) 
3. R. Rachamin, C. Wemple, E. Fridman, Ann. Nucl. Ener. 55, 194 (2006)

4. G. Kessedjian, G. Barreau, M. Aïche, B. Jurado, A. Bidaud, S. Czajkowski, D. Dassié, B. Haas, L. Mathieu, L. Tassan-Got, J.N. Wilson, F.-J. Hambsch, S. Oberstedt, I. AlMahamid, J. Floyd, W. Lukens, D. Shuh, Phys. Rev. C 85, 044613

5. Y. Naitou, Y. Watanabe, S. Hirayama, M. Hayashi, A. Prokofiev, A. Hjalmarsson, S. Pomp, P. Andersson, R. Bevilacqua, C. Gustavsson, M. Österlund, V. Simutkin, H. Sjöstrand, M. Tesinsky, U. Tippawan, Journ. of Kor. Phys. Soc. 59, No. 2 (2011)

6. K. Asai, N. Naoi, T. Iguchi, K. Watanabe, J. Kawarabayashi, T. Nishitani, J. Nucl. Sci. and Tech. 43, No 4 (2006)

7. R. Beyer, E. Grosse, K. Heidel, J. Hutsch, A.R. Junghans, J. Klug, D. Légrády, R. Nolte, S. Röttger, M. Sobiella, A. Wagner, Nucl. Instr. and Meth. A 575 (2007)

8. A. Donzella, M. Barbui, F. Bocci, G. Bonomi, M. Cinausero, D. Fabris, A. Fontana, E. Giroletti, M. Lunardon, S. Moretto, G. Nebbia, M.M. Necchi, S. Pesente, G. Prete, V. Rizzi, G. Viesti, A. Zenoni, Nucl. Instr. and Meth. A 613 (2010)

9. J. Hassard, J.H. Liu, R. Mongkolnavin, D. Colling, Nucl. Instr. and Meth. A 416 (1998)

10. C. Cazzaniga, M.Rebai, M. Tardocchi, G.Croci, M. Nocente, S.Ansell, C.D. Frost, G. Gorini, Prog. Theor. Exp. Phys., 073H01 (2015)

11. R. Babut, V. Gressier, J. Instr. in Nucl. Sci. and Tech. 2 P01005 (2007)

12. J. Taforeau, I. Schaefer, S. Higueret, D. Husson, L. Lebreton, Prog. Nucl. Sci. and Tech. Vol 4 (2014)

13. M.A. Kovash, B. Daub, J. French, V. Henzl, K. Shoniyozov, J.L. Matthews, Z. Miller, H. Yang, Proc. of conf. Adv. in Nucl. Inst. Meas. Meth. and Appl. (2011) 
